\title{
On Taxed Matrix Games and Changes in the Expected Transfer
}

\author{
Ingo Althöfer and Marlis Bärthel \\ Fakultät für Mathematik und Informatik, Friedrich-Schiller-Universität Jena, Ernst-Abbe-Platz 2, 07743 Jena, Germany \\ Correspondence should be addressed to Marlis Bärthel; marlis.baerthel@uni-jena.de
}

Received 19 May 2014; Revised 11 August 2014; Accepted 13 August 2014; Published 31 August 2014

Academic Editor: X. Henry Wang

Copyright (C) 2014 I. Althöfer and M. Bärthel. This is an open access article distributed under the Creative Commons Attribution License, which permits unrestricted use, distribution, and reproduction in any medium, provided the original work is properly cited.

In gambling scenarios the introduction of taxes may affect playing behavior and the transferred monetary volume. Using a game theoretic approach, we ask the following: How does the transferred monetary volume change when the winner has to pay a tax proportional to her win? In this paper we therefore introduce a new parameter: the expected transfer. For a zerosum matrix game with payoff matrix $A$ and mixed strategies $p$ and $q$ of the two players it is defined by $\operatorname{ET}(A ; p, q)=\sum \sum p_{i} q_{j}\left|a_{i j}\right|$. Surprisingly, it turns out that for small fair matrix games higher tax rates lead to an increased expected transfer. This phenomenon occurs also in analogous situations with tax on the loser, bonus for the winner, or bonus for the loser. Higher tax or bonus rates lead to overproportional expected revenues for the tax authority or overproportional expected expenses for the grant authority, respectively.

\section{Introduction}

We analyze how taxes change the character of a fair zerosum matrix game $\Gamma$ with payoff matrix $A=\left(a_{i j}\right)$. For mixed strategies $p$ and $q$ of the two players Max and Min, the expected value of $\Gamma$ with respect to $(p, q)$ is $v=v(\Gamma ; p, q)=$ $\sum \sum p_{i} q_{j} a_{i j}$. Sometimes one may not be interested in this value but instead in the expected transfer of $(\Gamma ; p, q)$. We define this new parameter by $\operatorname{ET}(\Gamma ; p, q)=\sum \sum p_{i} q_{j}\left|a_{i j}\right|$. The only difference between value and transfer is that in the payoff case the true $a_{i j}$ are taken, and in the transfer case the absolute values $\left|a_{i j}\right|$. Regarding the matrix entries as monetary units that are transferred from one player to the other (where the sign determines the direction), the expected transfer expresses how many units are transferred in the average, independent of the direction. This new parameter is an interesting one as it provides information about the player's behavior. The expected transfer is connected to the average stakes of the players. The higher the expected transfer, the higher the average stakes. Moreover, the expected transfer is an interesting parameter for tax authorities, as the expected tax revenue is directly coupled to it.

When $\Gamma$ is a matrix game with unique optimal strategies $\bar{p}$ and $\bar{q}$, we shortly write $\operatorname{ET}(\Gamma)=\operatorname{ET}(\Gamma ; \bar{p}, \bar{q})$. We investigate how $\operatorname{ET}(\Gamma)$ changes when the game $\Gamma$ is changed in a certain way. Our basic model assumes a "winner tax," say with $x=$ $10 \%$ rate. When row $i$ and column $j$ are played, then $\left|a_{i j}\right|$ is transferred. For $a_{i j}>0$ the winner tax means that player Max receives only $0.90 \cdot a_{i j}$, whereas player Min has to pay the full $a_{i j}$. For $a_{i j}<0$ player Max has to pay the full $\left|a_{i j}\right|$, but Min gets only $0.90 \cdot\left|a_{i j}\right|$. For $a_{i j}=0$ nothing at all is transferred and no taxes are taken from the players. We denote the new game by $\Gamma_{\mathrm{WiT}}(x)$, where the index "WiT" is short for "Winner Tax."

Introducing such a tax changes the character of the game. It is no longer zerosum, but a bimatrix game. Bimatrix games have one unpleasant property: there may be several equilibria, with different expected payoffs and transfers. However, at least in the case, where also the taxed game has a unique equilibrium, one may compare the expected transfers of $\Gamma_{\mathrm{WiT}}(x)$ and $\Gamma$. We do this comparison with respect to the original $\left|a_{i j}\right|$. So, when, for instance, in the taxed game, player Max gets $(1-x) \cdot a$ and Min has to pay $1 \cdot a$, then we count this as transfer of size $1 \cdot a$. That is, our transfer means "transfer before tax."

Naive common sense might suggest that such a winner tax should discourage high stakes, resulting in a smaller expected transfer. But analysis tells a different story, at least 
for small, fair matrix games. Often, a winner tax increases the expected transfer. In some special cases, namely, fair $2 \times 2$ matrix games and symmetric $3 \times 3$ matrix games, for every nondegenerate matrix game $\Gamma$, the tax leads to an increased expected transfer. For these cases we even prove a stronger statement: the higher the tax (until $x=100 \%$ ), the higher the expected transfer in the taxed game.

Analogously to the scenario with winner tax (called (a)), three other scenarios of "transfer manipulation" may be investigated.

(b) Loser Tax. The loser has to pay $(1+x) \cdot\left|a_{i j}\right|$ instead of simply $\left|a_{i j}\right|$; the winner gets $\left|a_{i j}\right|$.

(c) Winner Bonus. The winner gets $(1+x) \cdot\left|a_{i j}\right|$ instead of simply $\left|a_{i j}\right|$; the loser has to pay $\left|a_{i j}\right|$.

(d) Loser Bonus. The loser has to pay only $(1-x) \cdot\left|a_{i j}\right|$ instead of $\left|a_{i j}\right|$; the winner gets $\left|a_{i j}\right|$.

For fair $2 \times 2$ and symmetric $3 \times 3$ matrix games, all four scenarios lead to larger expected transfers for increasing rates $x$. Higher tax rates lead to overproportional expected revenues for the tax authority in (a) and (b). Analogously, higher bonus rates lead to overproportional expected expenses for the grant authority in (c) and (d). Moreover, for fixed rate $x \in[0,1)$, it surprisingly turns out that, for these games, the changes of the expected transfers in (a) and (d) are identical. Analogously, the changes of the expected transfers in (b) and (c) are identical. Here, furthermore, the (a, d)-increase is never smaller than the (b, c)-increase.

From our point of view the content of this paper should be understood as a new approach in fundamental research about playing behavior under taxation and its impacts on transfer activities. Allowing a courageous interpretation, the results may play a role in the field of gambling, when, for example, taxes are introduced in casinos, in betting business, on the stock market, and so forth. For the tax authorities (e.g., the state, an online-platform provider, etc.) in these relevant economic applications it is not important who of the participating players has which payoff. Instead the transferred monetary volume is interesting, because it determines resulting tax revenues.

However, our findings do not directly tie in with the existing body of literature or ought to be understood as an intent to represent a concrete practical case. We consider our main achievement in finding the model and obtaining the statement. Nevertheless, we refer to some cross-references in scientific work and possible applications in Section 2.

Subsequently, the paper is organized as follows. Section 3 includes preliminaries and basic definitions. For better understanding, we give a detailed example. Some readers may prefer to start their course through Section 3 with the example. The main result together with the basic ideas of its proof is presented in Section 4 . We conclude with a discussion and some open problems in Section 5.

\section{Related Scientific Work and Possible Applications}

The harvest of an extensive literature research on related work to our model was rather limited. In the classroom book Finite Mathematics by Waner and Costenoble [1, p.217] nonzerosum games are introduced, with the comment "An example $[\cdots]$ would be one in which the government taxed the earnings of the winner. In that case the winner's gain would be smaller than the loser's loss." Waner and Costenoble did no further elaboration on this remark. We did not find other studies of taxed matrix games in the literature. Existing analysis of bimatrix games with limited rank (cf. [2]) does not cover our investigations.

The concept of utility functions and risk aversion is an important object of investigation in game theory and economics (cf. [3, 4] for basic work on the topic). Our definitions of the four manipulation scenarios could alternatively be understood as risk averse behavior (with concave utility function by introducing winner or loser taxes) or risk loving behavior (with convex utility function by introducing winner or loser bonuses) of the two players. There are studies that examine the effect of risk aversion on the expected payoff in bimatrix games. Both, Berden and Peters [5], and Engelmann and Steiner [6] establish under which conditions one player's risk aversion in $2 \times 2$ bimatrix games is beneficial. They suggest that it is difficult to obtain results for the completely general case with larger matrices. Moreover, in their model starting with a zerosum matrix game would not be very interesting, as risk aversion or loving then has no effect at all on the expected payoffs.

In comparison to Berden and Peters [5] or Engelmann and Steiner [6], our model

(1) considers risk neutral utility functions,

(2) focuses on effects concerning the expected transfer,

(3) concentrates on fair zerosum matrix games.

We explicitly consider the four introduced scenarios (winner tax, loser tax, winner bonus, and loser bonus) as interventions from outside. Before discussing some possible implications and applications of our model in the following, it should be remarked that we do this in a very interpretative way.

A possible implication concerns stock and exchange markets. How could a financial transaction tax (e.g., a Tobin tax [7] or some others) change the behavior of the agents in the market? Of course, high-speed trading would no longer be attractive. But, when looking at "normally slow" agents, would they act more aggressively by putting higher stakes, resulting in higher transfers?

S. Hiss in a discussion immediately pointed out that bonuses for successful traders are common. Of course, situations with some sort of loser bonuses (= loser compensation) are frequent, for instance, when a state saves a bank that would otherwise go bankrupt. There even exist situations with loser taxes. That is, either directly, when for instance a loss in transaction carries the risk to lose a costumer or even the job. Or on a psychological level, when for instance an unsuccessful agents becomes depressive, what may lead 
to an additional drop in her payoff function. So, all four scenarios of payoff manipulation (winner tax, winner bonus, loser bonus, and loser tax) exist in the world of financial markets.

Implications in classical gambling situations, such as Poker, might be more apparent. Some variants of Casino Poker have become very popular since the 1990s. There has been theoretical work on the two-player zerosum scenario, starting already with von Neumann in his seminal paper from 1928 [8]. Modern approaches, for instance, the theoretical work by Koller et al. [9] and the computational approach by Billings et al. [10], give new insights. But it is generally accepted that Poker is much too complicated for a full analysis. Nevertheless, we would ask the following. How do winner/loser taxes/bonuses change the optimal strategies and the average height of their stakes?

There might be interesting differences in theoretical and real-life effects as well. What change in player behavior do we see in real-world games, for example, in the Internet Poker servers with different types and amounts at stake? Or what about actors participating in online betting portals where profit taxes are collected?

\section{Preliminaries and a Detailed Example}

In our model, taxes change a zerosum matrix game into a bimatrix game. Therefore we start with some preliminaries on bimatrix games.

Let $\Gamma=(A, B)$ be a bimatrix game, where $A=\left(a_{i j}\right) \epsilon$ $\mathbb{R}^{m \times n}, B=\left(b_{i j}\right) \in \mathbb{R}^{m \times n}$ are the payoff matrices for player 1 and player 2, respectively. If $\Gamma$ is a zerosum game (i.e., $A=-B$ ), we call it a matrix game. The matrix game is symmetric if in addition $A=-A^{\top}$ holds. A mixed strategy for player 1 is a vector $p \in \mathbb{R}^{m}$ of probabilities, where $p_{i}$ represents the probability of playing row $i$. Therefore the strategy set for player 1 is $S_{m}=\left\{p=\left(p_{1}, \ldots, p_{m}\right)^{\top} \mid \sum_{i=1}^{m} p_{i}=1, p_{i} \in[0,1]\right.$ for $i \in\{1, \ldots, m\}\}$. Similarly, player 2 may choose any mixed strategy $q \in S_{n}$. The support of a strategy $p \in S_{m}$ is the set $\operatorname{supp}(p)=\left\{i \in\{1, \ldots, m\} \mid p_{i}>0\right\}$. A strategy of support size 1 is called pure strategy.

We assume that both players have full information about the two payoff matrices and want to maximize only their own expected payoffs (under risk neutral utility functions). Nash [11] defined equilibrium situations, in which neither player gains by unilateral deviation from her equilibrium strategy. A pair of mixed strategies $\left(\bar{p} \in S_{m}, \bar{q} \in S_{n}\right)$ is a Nash equilibrium, if $\bar{p}$ and $\bar{q}$ are best responses to each other, that means if $p^{\top} A \bar{q} \leq \bar{p}^{\top} A \bar{q}$ for all $p \in S_{m}$ and $\bar{p}^{\top} B q \leq \bar{p}^{\top} B \bar{q}$ for all $q \in S_{n}$.

Nash proved the existence of at least one such equilibrium for any bimatrix game $\Gamma=(A, B)$. Bimatrix games have one unpleasant feature: often there does not exist a unique Nash equilibrium. To avoid some of the complications from this phenomenon, we restrict ourselves to the class of nondegenerate bimatrix games (cf., e.g., Avis et al. [12, p.13]). A bimatrix game $\Gamma=(A, B)$ is called nondegenerate if no mixed strategy with support of size $k$ has more than $k$ pure best responses.
The set of degenerate bimatrix games has measure zero. Moreover, it is known that every nondegenerate matrix game has exactly one Nash equilibrium. For bimatrix games in general, this is not true. However, Lemke and Howson [13] proved that each nondegenerate bimatrix game has an odd (in particular a finite) number of Nash equilibria.

Consider a nondegenerate matrix game $\Gamma=(A,-A)$ with equilibrium $(\bar{p}, \bar{q})$. As usual (cf. [3]), we define the value of the game by $\bar{v}(\Gamma):=\sum \sum \bar{p}_{i} \bar{q}_{j} a_{i j}$. We call $\Gamma$ a fair game if $\bar{v}=0$. Now we define the expected transfers of the original game $\Gamma$ and the manipulated game $\Gamma_{\mathrm{WiT}}(x)$ that results from charging a "winner tax" with tax rate $x \in[0,1)$.

Definition 1 (expected transfer). The expected transfer of a matrix game $\Gamma=(A,-A)$ with unique Nash equilibrium $(\bar{p}, \bar{q})$ is given by

$$
\operatorname{ET}(\Gamma):=\sum_{i=1}^{m} \sum_{j=1}^{n} \bar{p}_{i} \bar{q}_{j}\left|a_{i j}\right| .
$$

If the corresponding bimatrix game $\Gamma_{\mathrm{WiT}}(x)=(A(x), B(x))$ has a unique Nash equilibrium $(\bar{p}(x), \bar{q}(x))$, then the expected transfer of the manipulated game is

$$
\operatorname{ET}\left(\Gamma_{\mathrm{WiT}}(x)\right):=\sum_{i=1}^{m} \sum_{j=1}^{n} \bar{p}(x)_{i} \bar{q}(x)_{j}\left|a_{i j}\right| .
$$

The expected transfers of the manipulated games $\Gamma_{\mathrm{LoT}}(x)$, $\Gamma_{\mathrm{WiB}}(x)$, and $\Gamma_{\mathrm{LoB}}(x)$ in the three other scenarios (loser tax, winner bonus, and loser bonus) are defined analogously.

Example 2. We analyze the impact of taxes or bonuses on the expected transfer. As an example we look at the nondegenerate $2 \times 2$ matrix game $\Gamma=(A,-A)$ with

$$
A=\left(\begin{array}{cc}
1 & -2 \\
-3 & 6
\end{array}\right)
$$

This game has the unique equilibrium $(\bar{p}, \bar{q})=\left((3 / 4,1 / 4)^{\top}\right.$, $\left.(2 / 3,1 / 3)^{\top}\right)$. The value of the game is $\bar{v}=\bar{p}^{\top} A \bar{q}=0$, so it is a fair game. With Definition 1, we can compute the expected transfer of $\Gamma$ :

$$
\begin{aligned}
\operatorname{ET}(\Gamma) & =\left(\begin{array}{ll}
\frac{3}{4} & \frac{1}{4}
\end{array}\right)\left(\begin{array}{ll}
1 & 2 \\
3 & 6
\end{array}\right)\left(\begin{array}{c}
\frac{2}{3} \\
\frac{1}{3}
\end{array}\right) \\
& =\frac{3 \cdot 2 \cdot 1+3 \cdot 1 \cdot 2+1 \cdot 2 \cdot 3+1 \cdot 1 \cdot 6}{12}=2 .
\end{aligned}
$$

Now we assume that a third party enters the scene and collects a game tax, say $10 \%$ of the transfer, to be payed by the winner. This diminishes the positive entries in the payoff matrices for both players. Concretely, the game in the example becomes the bimatrix game $\Gamma_{\mathrm{WiT}}(0.1)=(A(0.1), B(0.1))$ with

$$
A(0.1)=\left(\begin{array}{ll}
0.9 & -2 \\
-3 & 5.4
\end{array}\right), \quad B(0.1)=\left(\begin{array}{cc}
-1 & 1.8 \\
2.7 & -6
\end{array}\right)
$$


This taxed game has a unique Nash equilibrium too; namely, $(\bar{p}(0.1), \bar{q}(0.1))=\left((87 / 115,28 / 115)^{\top},(74 / 113,39 / 113)^{\top}\right)$. The expected payoffs of the two players in this new situation are negative: $v_{1}(0.1)=\bar{p}(0.1)^{\top} A(0.1) \bar{q}(0.1)=-57 / 565 \approx$ -0.1009 and $v_{2}(0.1)=\bar{p}(0.1)^{\top} B(0.1) \bar{q}(0.1)=-57 / 575 \approx$ -0.0991 . As specified in Definition 1 , the expected transfer is computed with the original payoffs:

$$
\begin{aligned}
\operatorname{ET}\left(\Gamma_{\mathrm{WiT}}(0.1)\right) & =\left(\begin{array}{ll}
\frac{87}{115} & \frac{28}{115}
\end{array}\right)\left(\begin{array}{ll}
1 & 2 \\
3 & 6
\end{array}\right)\left(\begin{array}{c}
\frac{74}{113} \\
\frac{39}{113}
\end{array}\right) \\
& =2+\frac{2}{12995} \approx 2.00015
\end{aligned}
$$

The expected transfer has increased. Admittedly, the increase is only a small one with about 2.00015 units instead of exactly 2. The expected tax revenue for the tax authority is given by $0.1 \cdot \operatorname{ET}\left(\Gamma_{\mathrm{WiT}}(0.1)\right)=12996 / 64975 \approx 0.2$. Note that the expected tax revenue for the tax authority corresponds to the aggregated expected losses of the two players: $-v_{1}(0.1)-$ $v_{2}(0.1)=12996 / 64975$.

For the same matrix $A$, we now look at a general tax rate $x \in[0,1)$. The payoff matrices in the bimatrix game $\Gamma_{\mathrm{WiT}}(x)=$ $(A(x), B(x))$ are

$$
\begin{aligned}
& A(x)=\left(\begin{array}{cc}
(1-x) \cdot 1 & -2 \\
-3 & (1-x) \cdot 6
\end{array}\right) \\
& B(x)=\left(\begin{array}{cc}
-1 & (1-x) \cdot 2 \\
(1-x) \cdot 3 & -6
\end{array}\right) .
\end{aligned}
$$

In this more general situation, the Nash equilibrium of the bimatrix game is still unique. Depending on the tax rate $x$, the equilibrium strategies are

$$
\begin{aligned}
& (\bar{p}(x), \bar{q}(x)) \\
& \quad=\left(\left(\frac{9-3 x}{12-5 x}, \frac{3-2 x}{12-5 x}\right)^{\top},\left(\frac{8-6 x}{12-7 x}, \frac{4-x}{12-7 x}\right)^{\top}\right) .
\end{aligned}
$$

The expected payoffs for the two players (both negative for $x \in(0,1))$ are

$$
\bar{v}_{1}(x)=\frac{6 x(x-2)}{12-7 x}, \quad \bar{v}_{2}(x)=\frac{6 x(x-2)}{12-5 x},
$$

and the expected transfer is

$$
\operatorname{ET}\left(\Gamma_{\mathrm{WiT}}(x)\right)=\frac{72(x-2)^{2}}{35 x^{2}-144 x+144} .
$$

This function is strictly increasing for $x \in[0,1)$. The expected tax revenues are directly coupled to the expected transfer. Here, they are given by

$$
\text { expected tax revenue }(\text { rate } x)=x \cdot \operatorname{ET}\left(\Gamma_{\mathrm{WiT}}(x)\right) \text {. }
$$

Higher winner tax rates lead to overproportional higher expected revenues for the tax authority, as

$$
\begin{aligned}
& \frac{\text { expected tax revenue }(\text { rate } 2 x)}{\text { expected tax revenue }(\text { rate } x)} \\
& =\frac{2 x \cdot \operatorname{ET}\left(\Gamma_{\mathrm{WiT}}(2 x)\right)}{x \cdot \operatorname{ET}\left(\Gamma_{\mathrm{WiT}}(x)\right)} \geq \frac{2 x}{x} \cdot 1=2 .
\end{aligned}
$$

Calculation and argumentation for the other scenarios (LoT, $\mathrm{WiB}$, and $\mathrm{LoB}$ ) work analogously. In the bonus scenarios there are no revenues, but expenses for the grant authority. Figure 1(a) shows the expected transfers for the example game and all tax or bonus rates $x \in[0,1)$. Figure $1(\mathrm{~b})$ shows the resulting expected tax revenues or expected bonus expenses, respectively.

These phenomena are not an artifact of the special $2 \times$ 2 game with matrix $A$ from above, as shown by Theorem 3 in Section 4. Resulting effects (represented by Figure 1) occur for all nondegenerate, fair $2 \times 2$ matrix games.

\section{Main Results}

Analyzing the changes in the expected transfer, we found the following result. All statements can clearly be related to the graph in Figure 1(a)

Theorem 3. Let $\Gamma=(A,-A)$ be a nondegenerate, fair $2 \times 2$ matrix game. For every tax or bonus rate $x \in[0,1)$, the following six statements hold.

(i) The bimatrix games $\Gamma_{W i T}(x), \Gamma_{L o T}(x), \Gamma_{W i B}(x)$, and $\Gamma_{L o B}(x)$ each have exactly one Nash equilibrium.

(ii) $\operatorname{ET}\left(\Gamma_{W i T}(x)\right)=E T\left(\Gamma_{L o B}(x)\right)$.

(iii) $\operatorname{ET}\left(\Gamma_{L o T}(x)\right)=E T\left(\Gamma_{W i B}(x)\right)$.

(iv) $\operatorname{ET}\left(\Gamma_{W i T}(x)\right)$ is either strictly increasing in $x$ or constant.

(v) $E T\left(\Gamma_{L o T}(x)\right)$ is either strictly increasing in $x$ or constant.

(vi) $E T\left(\Gamma_{W i T}(x)\right) \geq E T\left(\Gamma_{L o T}(x)\right) \geq E T(\Gamma)$.

Proof. An arbitrary $2 \times 2$ matrix game has four parameters. Normally these are given by the four entries in the matrix $A=$ $\left(\begin{array}{ll}a_{11} & a_{12} \\ a_{21} & a_{22}\end{array}\right)$.

With a well-known fact about strategically equivalent games (cf., e.g., Szép and Forgó [14, p.106]), we can assume that the first entry $a_{11}$ is equal to 1 . The bimatrix games $\Gamma=$ $(A, B)$ and $\Gamma^{\prime}=\left(c A+d \mathbb{1}_{m \times n}, e B+f \mathbb{1}_{m \times n}\right)$ have the same equilibria for all $c, d, e, f \in \mathbb{R}$ with $c, e>0$. (Here, $\mathbb{1}_{m \times n}$ denotes the $m \times n$ matrix with all entries equal to 1 .)

Let $\Gamma=(A,-A)$ be a nondegenerate, fair $2 \times 2$ matrix game. With the settings $\bar{v}=0$ and $a_{11}=1$, two of the four free parameters are already fixed.

As the equilibrium strategies in nondegenerate matrix and bimatrix games have supports of the same size (cf., e.g., [12, p.13]) we distinguish two cases, according to the support sizes of the equilibrium strategies. Games with pure equilibrium can be analyzed easily in a side case. As the 


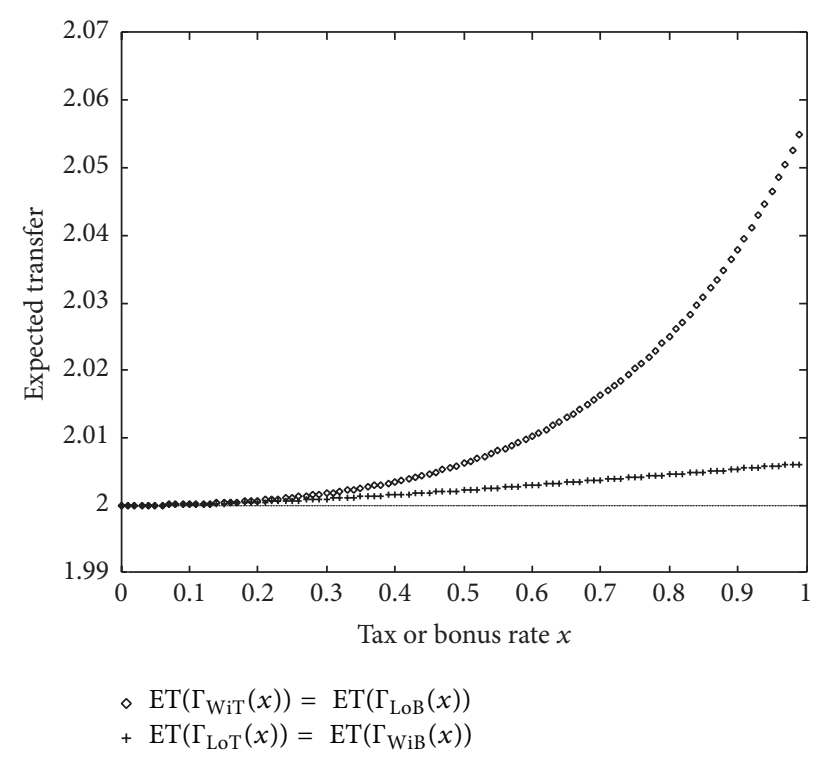

(a) Expected transfers

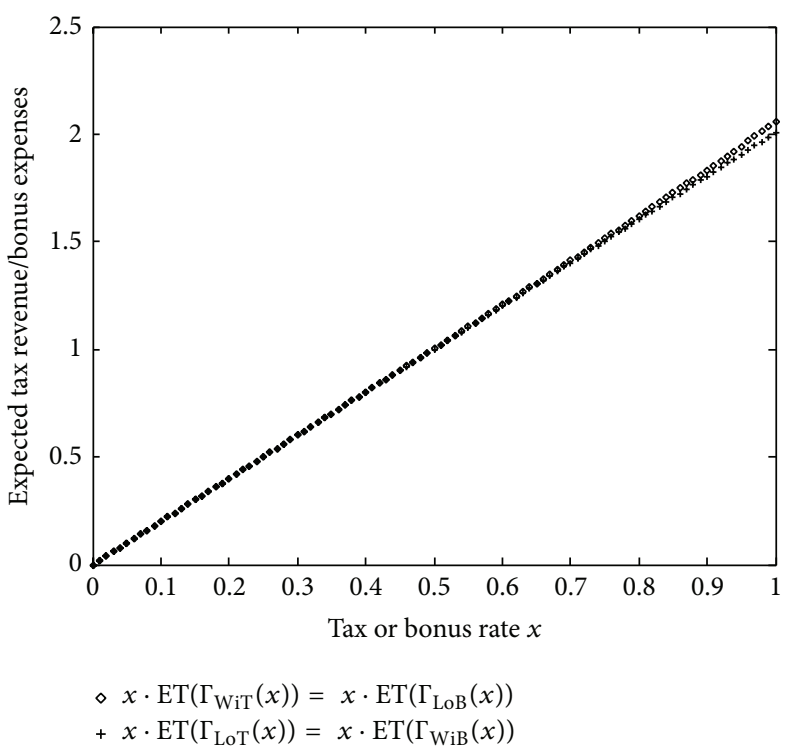

(b) Expected tax revenues or bonus expenses

FIGURE 1: Results for the example game and all four scenarios with tax or bonus rate $x \in[0,1)$.

manipulation rate $x$ is out of the interval $[0,1)$, the resulting bimatrix game (in any of the four scenarios) is nondegenerate with the same pure Nash equilibrium as in the original matrix game. (Note that the rate $x=1$ may provoke degeneracy and therefore is excluded here.) Since the value of the game is $\bar{v}(\Gamma)=0$, it turns out that the expected transfer then is 0 in all four scenarios for every tax or bonus rate $x \in[0,1)$.

More interesting is the main case, where the matrix game has a mixed equilibrium. Here, we define the remaining two (of the required four) parameters by declaring the players equilibrium strategies to be $\bar{p}=(p, 1-p)^{\top}$ and $\bar{q}=(q, 1-q)^{\top}$, respectively, with parameters $p, q \in(0,1)$. The matrix $A$ then must have the form

$$
A=\left(\begin{array}{cc}
1 & -\frac{q}{1-q} \\
-\frac{p}{1-p} & \frac{p q}{(1-p)(1-q)}
\end{array}\right) .
$$

We manipulate the entries of $A$ according to one of our four scenarios with rate $x \in[0,1)$. The resulting bimatrix game is nondegenerate with unique mixed equilibrium because of the persisting structure of signs. In fact, this holds for $x=1$, too.

It is possible to determine explicit expressions for the equilibrium strategies and the expected transfers in all four scenarios. Table 1 gives an overview of the corresponding results depending on the two parameters $p, q \in(0,1)$ and on the tax or bonus rate $x \in[0,1)$. The results in the table are also true for the rate $x=1$.

For fixed rate $x \in[0,1)$, the two identities $\operatorname{ET}\left(\Gamma_{\mathrm{WiT}}(x)\right)=$ $\mathrm{ET}\left(\Gamma_{\mathrm{LoB}}(x)\right)$ and $\operatorname{ET}\left(\Gamma_{\mathrm{LoT}}(x)\right)=\mathrm{ET}\left(\Gamma_{\mathrm{WiB}}(x)\right)$ follow immediately.
All other properties can be shown by some straightforward calculations. As an illustrative example, we prove the fact (iv) of Theorem 3: " $\mathrm{ET}\left(\Gamma_{\mathrm{WiT}}(x)\right)$ is either strictly increasing in $x$ or constant." The statement can be shown by demonstrating that the expression $\operatorname{ET}\left(\Gamma_{\mathrm{WiT}}(x)\right)$, as a function in $x \in[0,1)$, is monotonically increasing. The derivative is

$$
\begin{aligned}
& \frac{d \mathrm{ET}\left(\Gamma_{\mathrm{WiT}}(x)\right)}{d x} \\
& =\frac{d \mathrm{ET}\left(\Gamma_{\mathrm{LoB}}(x)\right)}{d x} \\
& =\frac{-p q x(x-2)(4(p+q-2 p q)(p+q-2 p q-1)+1)}{\left(x^{2}(p+q-2 p q)(p+q-2 p q-1)+x-1\right)^{2}} .
\end{aligned}
$$

The expected transfer $\operatorname{ET}\left(\Gamma_{\mathrm{WiT}}(x)\right)=\mathrm{ET}\left(\Gamma_{\mathrm{LoB}}(x)\right)$ is monotonically increasing over $x \in[0,1)$, if and only if

$$
\begin{aligned}
& \frac{d \mathrm{ET}\left(\Gamma_{\mathrm{WiT}}(x)\right)}{d x} \geq 0 \\
& \Longleftrightarrow-p q x(x-2)(4(p+q-2 p q) \\
& \quad \times(p+q-2 p q-1)+1) \geq 0 \\
& \Longleftrightarrow(p+q-2 p q)((p+q-2 p q)-1) \geq-\frac{1}{4} .
\end{aligned}
$$

The last inequality holds, as for $p, q \in(0,1)$ the term $(p+q-$ $2 p q)$ has a codomain of $(0,1)$ and the function $f(z)=z(z-$ $1)$, with domain $(0,1)$, reaches its minimum in $(z, f(z))=$ $(1 / 2,-1 / 4)$. 
TABLE 1: Equilibrium strategies and expected transfers in all four scenarios (with tax or bonus rate $x \in[0,1)$ ) for nondegenerate, fair $2 \times 2$ matrix games with mixed equilibrium.

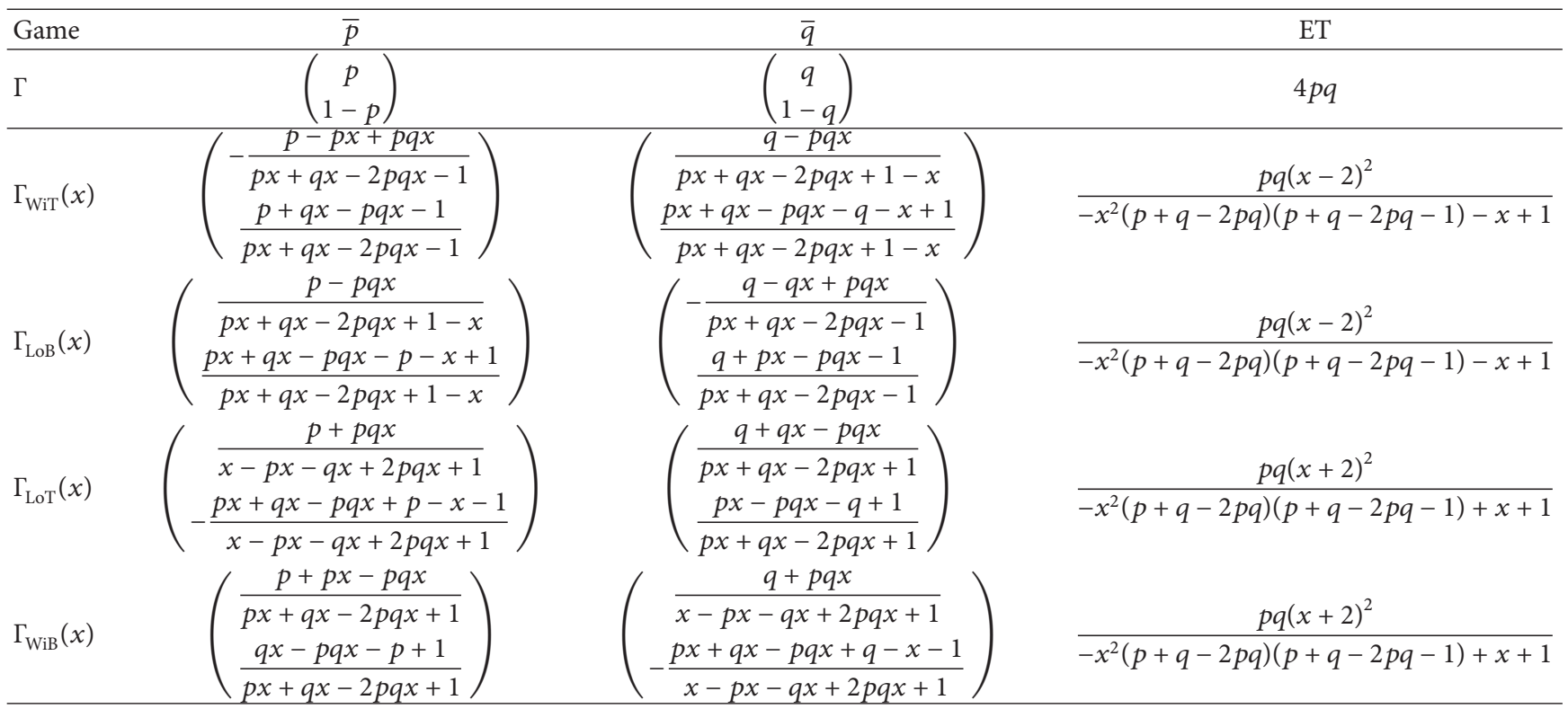

In addition, the last inequality reveals another interesting aspect. Only in case of $p=1 / 2$ or $q=1 / 2$, the first derivative is equal to zero for all $x \in[0,1)$. That means, among the fair games with mixed equilibrium strategies, only nondegenerate multiples of the matrix games $\Gamma=(A,-A)$ with

$$
A \in\left\{\left(\begin{array}{cc}
1 & -1 \\
-1 & 1
\end{array}\right),\left(\begin{array}{cc}
1 & -\lambda \\
-1 & \lambda
\end{array}\right),\left(\begin{array}{cc}
1 & -1 \\
-\lambda & \lambda
\end{array}\right)\right\}, \quad \lambda>0,
$$

have a constant expected transfer for all tax or bonus rates $x \in[0,1)$.

To prove the missing inequality of Theorem 3,

$$
\begin{aligned}
\operatorname{ET}\left(\Gamma_{\mathrm{WiT}}(x)\right) & =\operatorname{ET}\left(\Gamma_{\mathrm{LoB}}(x)\right) \\
& \geq \operatorname{ET}\left(\Gamma_{\mathrm{LoT}}(x)\right)=\operatorname{ET}\left(\Gamma_{\mathrm{WiB}}(x)\right),
\end{aligned}
$$

we show

$$
\begin{aligned}
& \operatorname{ET}\left(\Gamma_{\mathrm{WiT}}(x)\right)-\operatorname{ET}\left(\Gamma_{\mathrm{LoT}}(x)\right) \\
& =\frac{-2 p q x^{3}(2 p-1)^{2}(2 q-1)^{2}}{\left((p x+q x-2 p q x)^{2}-1\right)\left(1-(p x+q x-2 p q x-x)^{2}\right)}
\end{aligned}
$$

$$
\geq 0 \text {. }
$$

This is true, since

(i) $-2 p q x^{3}(2 p-1)^{2}(2 q-1)^{2} \leq 0$ obviously,

(ii) $(p x+q x-2 p q x)^{2}-1=\underbrace{x^{2}}_{\in(0,1)} \underbrace{(p+q-2 p q)^{2}}_{\in(0,1)}-1<0$,

(iii) $1-(p x+q x-2 p q x-x)^{2}=1-\underbrace{x^{2}}_{\in(0,1)} \underbrace{(p+q-2 p q-1)^{2}}_{\in(0,1)}>$ 0.
Remark 4. As observed in the example, taxes lead to expected losses of both players in the more general case of any nondegenerate, fair $2 \times 2$ matrix game, too. Analogously, bonuses lead to expected profits of both players. Straightforward calculation shows that, for all nondegenerate, fair $2 \times 2$ matrix games, the expected tax revenues exactly correspond to the aggregated expected losses of the two players. This holds for every tax rate $x$ in each of the tax scenarios. That is, for example, in the winner tax scenario,

$$
x \cdot \operatorname{ET}\left(\Gamma_{\mathrm{WiT}}(x)\right)=-v_{1}(x)-v_{2}(x) .
$$

Analogously, the expected bonus expenses correspond exactly to the aggregated expected profits of the two players for every bonus rate $x$ in the bonus scenarios.

Remark 5. For all nondegenerate, fair $2 \times 2$ matrix games, higher tax rates lead to overproportional higher expected tax revenues in the tax scenarios. The argumentation works exactly as shown for the example game. Analogously, higher bonus rates lead to overproportional higher expected bonus expenses in the bonus scenarios.

We have shown all statements of Theorem 3 to be true for any nondegenerate, fair $2 \times 2$ matrix game. For fair $3 \times 3$ matrix games in general, they are not true, but results do hold for symmetric $3 \times 3$ matrix games, too. Remarks 4 and 5 apply to all nondegenerate, symmetric $3 \times 3$ matrix games as well.

Theorem 6. Let $\Gamma=(A,-A)$ be a nondegenerate, symmetric $3 \times 3$ matrix game. For every tax or bonus rate $x \in[0,1)$, the same six statements (i)-(vi) as in Theorem 3 hold.

Proof. Demonstrating the statements for nondegenerate, symmetric $3 \times 3$ matrix games basically works in the same way as for fair $2 \times 2$ matrix games. The case with pure equilibrium 
strategies once again leads to constant expected transfers in all four scenarios.

For the nondegenerate case with mixed equilibrium strategies we start with the matrix

$$
A=\left(\begin{array}{ccc}
0 & 1 & -a_{13} \\
-1 & 0 & a_{23} \\
a_{13} & -a_{23} & 0
\end{array}\right)
$$

with $a_{13}>0$ and $a_{23}>0$. Statements (i) $-($ vi) can again be proved by straightforward, but lengthy calculations, resulting in case-by-case analyses and parametrical discussions (in $a_{13}$, $a_{23}$, and $x$ ). Complete proofs can be found in Althöfer and Bärthel $[15$, p. $21 \mathrm{ff}]$.

Remark 7. It is possible to specify the statements in Theorems 3 and 6 , respectively. We can list those matrix games with a constant expected transfer in all four scenarios. For every tax or bonus rate $x \in(0,1)$, the games described below are the only ones that satisfy the whole chain of equalities $\operatorname{ET}(\Gamma)=$ $\mathrm{ET}\left(\Gamma_{\mathrm{WiT}}(x)\right)=\mathrm{ET}\left(\Gamma_{\mathrm{LoB}}(x)\right)=\mathrm{ET}\left(\Gamma_{\mathrm{LoT}}(x)\right)=\mathrm{ET}\left(\Gamma_{\mathrm{WiB}}(x)\right)$.

Among the nondegenerate, fair $2 \times 2$ matrix games,

(i) every nondegenerate, fair $2 \times 2$ matrix game with pure equilibrium (i.e., both players chose with probability 1 one special row or column, resp.);

(ii) every nondegenerate multiple of a matrix game $\Gamma=$ $(A,-A)$ with

$$
A \in\left\{\left(\begin{array}{cc}
1 & -1 \\
-1 & 1
\end{array}\right),\left(\begin{array}{cc}
1 & -\lambda \\
-1 & \lambda
\end{array}\right),\left(\begin{array}{cc}
1 & -1 \\
-\lambda & \lambda
\end{array}\right)\right\}, \quad \lambda>0 .
$$

This set of matrix games has measure zero within the class of nondegenerate, fair $2 \times 2$ matrix games with completely mixed equilibrium strategies.

Among the nondegenerate, symmetric $3 \times 3$ matrix games,

(i) every nondegenerate symmetric $3 \times 3$ matrix game with pure equilibrium;

(ii) every nondegenerate multiple of the matrix game $\Gamma=$ $(A,-A)$ with

$$
A=\left(\begin{array}{ccc}
0 & 1 & -1 \\
-1 & 0 & 1 \\
1 & -1 & 0
\end{array}\right)
$$

This set of matrix games has measure zero within the class of nondegenerate, symmetric $3 \times 3$ matrix games with completely mixed equilibrium strategies.

For all other nondegenerate, fair $2 \times 2$ and nondegenerate, symmetric $3 \times 3$ matrix games $\Gamma$, the expected transfer in all four scenarios is strictly increasing in $x$ and the chain of inequalities in (iv) is a strict one:

$$
\begin{aligned}
\operatorname{ET}\left(\Gamma_{\mathrm{WiT}}(x)\right) & =\operatorname{ET}\left(\Gamma_{\mathrm{LoB}}(x)\right)>\operatorname{ET}\left(\Gamma_{\mathrm{LoT}}(x)\right) \\
& =\operatorname{ET}\left(\Gamma_{\mathrm{WiB}}(x)\right)>\operatorname{ET}(\Gamma) .
\end{aligned}
$$

Here, the clear structure for expected transfers in taxed matrix games (currently) ends. For nondegenerate, fair $3 \times$ 3 matrix games and for symmetric matrix games of larger size, our observed phenomena do not hold in every instance. Counterexamples can be given (cf. [15, p.27]) that reveal one interesting feature. Often, in these cases, the equilibrium strategies and thus the expected transfer need not be uniquely determined for some rates $x \in[0,1)$. Besides existing counterexamples, numerical experiments indicate an attenuation of the observed phenomena as the size of the matrix games increases (see [15, p.29 ff] for detailed information).

\section{Conclusion, Discussion, and Open Problems}

(i) For small matrix games, the introduction of taxes and bonuses often results in higher expected transfers. For two classes (nondegenerate, fair $2 \times 2$ and nondegenerate, symmetric $3 \times 3$ matrix games) we proved this phenomenon to hold in every instance.

(ii) Although our results hold only for small matrix games they are far more than just a little curiosity. Our model shows that one has to be careful in considering taxes as panacea to confine aggressive playing behavior. Moreover, we consider the limitation on small matrix games not as a too restrictive point, as situations of deciding among few opportunities do appear rather often, for example, in classical "either or," "yes or no," or "yes, no or neutral" noncooperative, two-player situations.

(iii) Although the scenarios with winner tax and loser bonus (or with loser tax and winner bonus, resp.) have exactly the same influence on the expected transfer of small matrix games, the equilibrium strategies typically differ. For the example game in Section 3, for instance, the equilibrium strategies after giving a bonus of $10 \%$ to the loser are $(\bar{p}(0.1), \bar{q}(0.1))=$ $\left((84 / 113,29 / 113)^{\top},(78 / 115,37 / 115)^{\top}\right)$. In the case of charging a $10 \%$ winner tax, the equilibrium strategies are $(\bar{p}(0.1), \bar{q}(0.1))=\left((87 / 115,28 / 115)^{\top}\right.$, $\left.(74 / 113,39 / 113)^{\top}\right)$.

(iv) Viewing the expected transfers for winner tax and winner bonus as two sides of the same function $\mathrm{ET}_{\text {Win }}(x)$ (taxes mean $x>0$; bonuses mean $x<$ 0 ), one realizes that this function $\operatorname{ET}_{\text {Win }}(x)$ is not only continuous in the whole interval $[-1,+1]$ but also smooth. In fact, $\mathrm{ET}_{\mathrm{Win}}(x)$ has a local minimum at $x=0$. The same argument applies to the sce -narios with taxes/bonuses for the loser and the corresponding expected transfers $\mathrm{ET}_{\text {Loss }}(x)$. An open question is as follows: do there exist nondegenerate, fair matrix games (of any size), where both $\operatorname{ET}_{\text {Win }}(x)$ and $\mathrm{ET}_{\text {Loss }}(x)$ have strict local maxima at rate $x=0$ ?

(v) Taxing the payoffs of the two players in originally fair matrix games leads to expected losses for both players (cf. Remark 4 in Section 4). One might argue that the players then would probably not play the game anymore. In the situation we describe, the players 
have to play the game and want to maximize their expected payoff, even if it is negative. Of course, work on zerosum games with value $v \neq 0$ or on constant sum games would be interesting, as well as the analysis of games with an option to pass.

(vi) A natural generalisation is to look at bimatrix games with taxes and bonuses and even at games (in normal form) for more than two players. In these scenarios "transfer" should be defined as the sum of the absolute values of the payoffs for the players. As done by Berden and Peters [5] and Engelmann and Steiner [6], one could start with an analysis of taxing only one player. Moreover, there may be a cross-connection to results by Bárány et al. [16], who prove that with huge probability large bimatrix games have equilibria with supports of size two. However, these "compact" equilibria need not be the most important ones.

(vii) When computing the equilibrium strategies in Section 3, we pointed out a well-known fact that is easily forgotten. Assume a bimatrix game with unique equilibrium in widely mixed strategies. Then for a player it is much more important to know the exact payoff matrix of the other player than to know his own one. In case of an $n \times n$ game with completely mixed strategies (all $p_{i}$ and $q_{j}>0$ ), her own payoff matrix has-despite its existence-no meaning for the computation of the probabilities in the optimal mixed strategy.

(viii) Bimatrix games are strange animals. They tend to have multiple equilibria, often with different values. Sensitivity analysis with small changes of parameters frequently leads to unexpected results. The increased expected transfer in case of taxes and bonuses on small, fair games is one of them.

\section{Conflict of Interests}

The authors declare that there is no conflict of interests regarding the publication of this paper.

\section{Acknowledgments}

Chrilly Donninger, Stefanie Hiss, Dietmar Wolz, and Wesley Turner gave their time for discussions and proofreading. Bernhard von Stengel was so kind to look in his "archive" for relevant papers. Special thanks go to the students Katharina Collatz and Christian Kubitza. The authors' students in Jena asked many critical questions. The authors thank Rahul Savani for his nice online bimatrix solver (http://banach.lse.ac.uk/form.html). Two anonymous referees gave constructive criticisms and helpful comments that improved the paper.

\section{References}

[1] S. Waner and S. R. Costenoble, Finite Mathematics, Brooks/ Cole, Boston, Mass, USA, 2007.
[2] J.-P. Beaud, “Antagonistic games," Mathématiques et Sciences Humaines, vol. 157, pp. 5-26, 2002.

[3] J. von Neumann and O. Morgenstern, Theory of Games and Economic Behaviour, Princeton University Press, Princeton, NJ, USA, 1944.

[4] J. W. Pratt, "Risk aversion in the small and in the large," Econometrica, vol. 32, no. 1-2, pp. 122-136, 1964.

[5] C. Berden and H. Peters, "On the effect of risk aversion in bimatrix games," Theory and Decision, vol. 60, no. 4, pp. 359370, 2006.

[6] D. Engelmann and J. Steiner, "The effects of risk preferences in mixed-strategy equilibria of $2 \times 2$ games," Games and Economic Behavior, vol. 60, no. 2, pp. 381-388, 2007.

[7] J. Tobin, "A proposal for international monetary reform," Eastern Economic Journal, vol. 4, no. 3-4, pp. 153-159, 1978.

[8] J. von Neumann, "Zur Theorie der Gesellschaftsspiele," Mathematische Annalen, vol. 100, no. 1, pp. 295-320, 1928.

[9] D. Koller, N. Megiddo, and B. Von Stengel, "Efficient computation of equilibria for extensive two-person games," Games and Economic Behavior, vol. 14, no. 2, pp. 247-259, 1996.

[10] D. Billings, N. Burch, A. Davidson et al., "Approximating gametheoretic optimal strategies for full-scale poker," in Proceedings of the 18th International Joint Conference on Artificial Intelligence (IJCAI '03), pp. 661-668, Acapulco, Mexico, August 2003.

[11] J. Nash, "Non-cooperative games," Annals of Mathematics, vol. 54, no. 2, pp. 286-295, 1951.

[12] D. Avis, G. D. Rosenberg, R. Savani, and B. von Stengel, "Enumeration of Nash equilibria for two-player games," Economic Theory, vol. 42, no. 1, pp. 9-37, 2009.

[13] C. E. Lemke and J. T. Howson, "Equilibrium points of bimatrix games," Journal of the Society for Industrial and Applied Mathematics, vol. 12, no. 2, pp. 413-423, 1964.

[14] J. Szép and F. Forgó, Introduction to the Theory of Games, Akadémiai Kiadó, Budapest, Hungary, 1985.

[15] I. Althöfer and M. Bärthel, "Taxed matrix games and changes in the expected transfer," Tech. Rep., Friedrich-SchillerUniversität Jena, Fakultät für Mathematik und Informatik, 2014, http://www.minet.uni-jena.de/preprints/althoefer_14/TaxedMatrixGames.pdf.

[16] I. Bárány, S. Vempala, and A. Vetta, "Nash equilibria in random games," Random Structures and Algorithms, vol. 31, no. 4, pp. 391-405, 2007. 


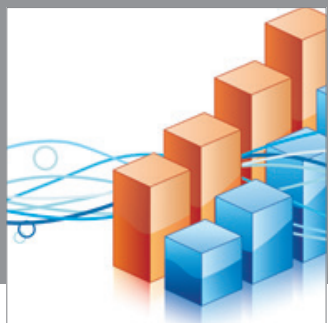

Advances in

Operations Research

mansans

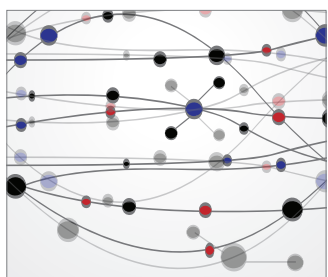

The Scientific World Journal
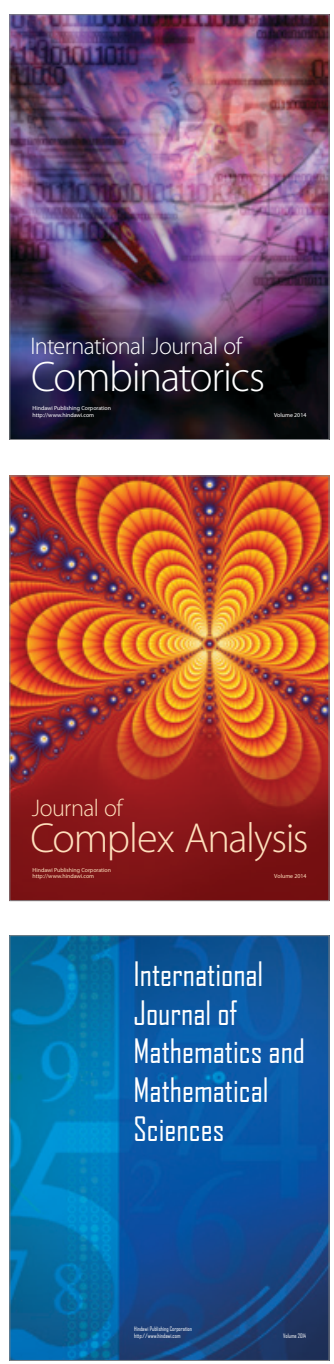
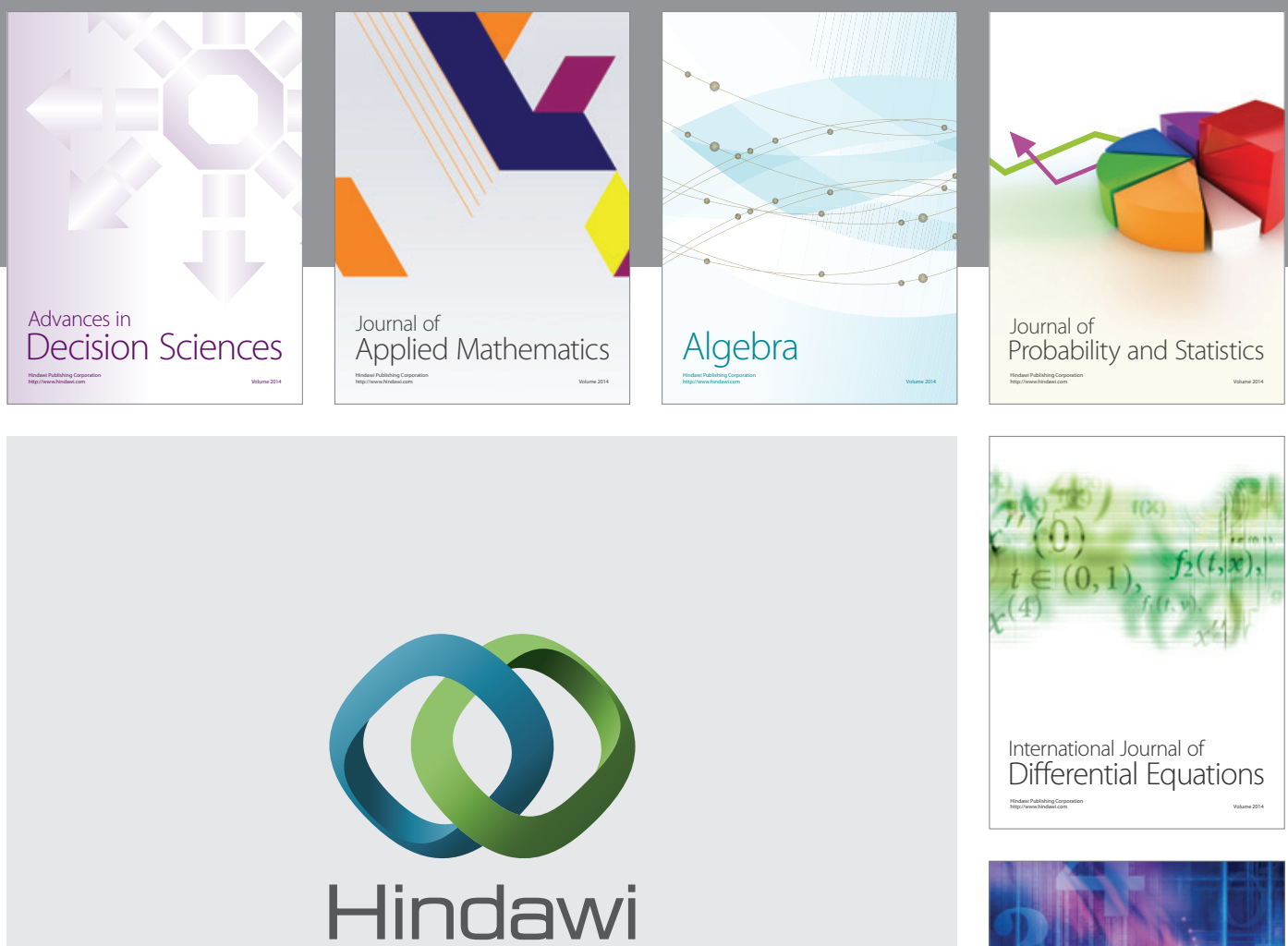

Submit your manuscripts at http://www.hindawi.com
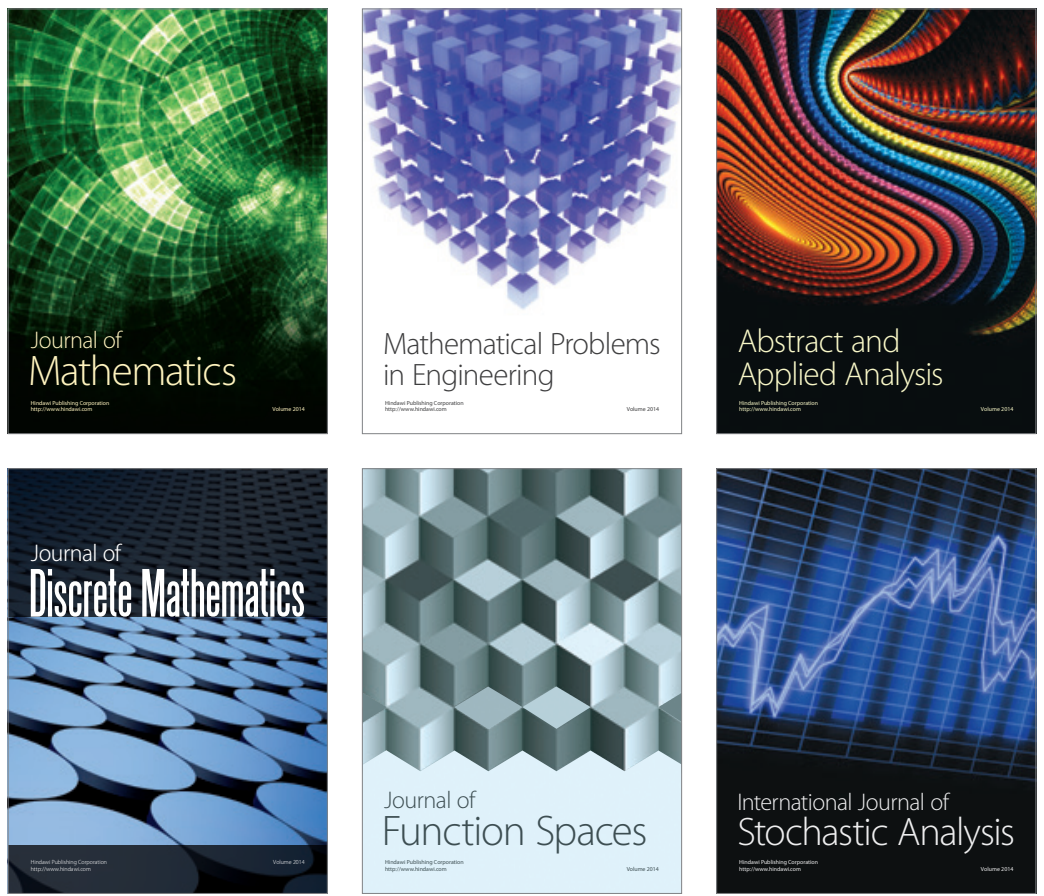

Journal of

Function Spaces

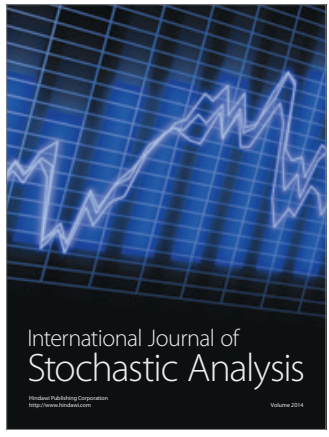

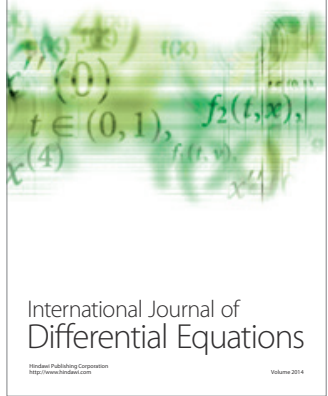
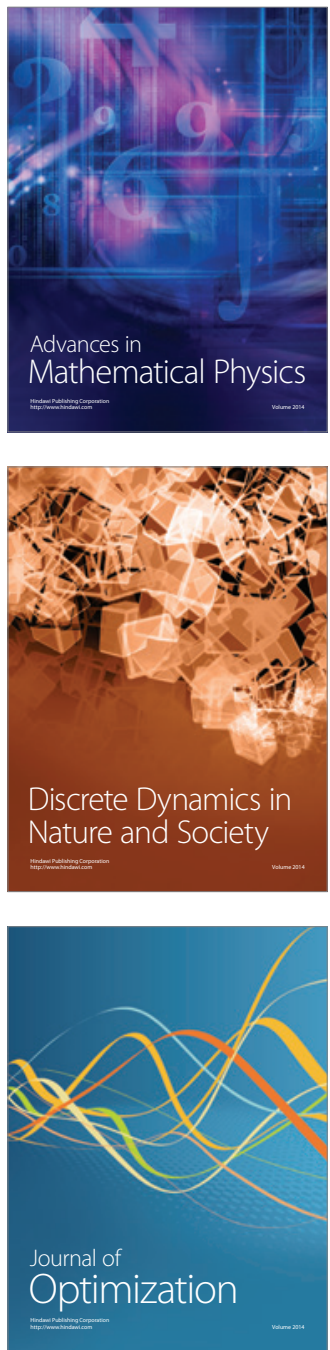